\title{
Preparation and characterization of gelatin sponge millispheres injectable through microcatheters
}

\author{
Noboru Yamashita' \\ Katsumi Saitou' \\ Akira Takagi' \\ Atsushi Maruyama² \\ 'Pharmaceutical Research \\ and Technology Labs, Institute \\ for Technology, Astellas Pharma Inc., \\ I 80 Ozumi, Yaizu-shi, Shizuoka-ken \\ 425-0072, Japan; ${ }^{2}$ Institute \\ for Materials Chemistry and \\ Engineering, Kyushu University, \\ 744-CEII Motooka, Nishi-ku, Fukuoka \\ 819-0395, Japan
}

\begin{abstract}
Objective: Millimeter size gelatin sponges are commonly used as an embolic agent for transcatheter arterial embolization (TAE). However the preparation of the fragments is troublesome and carries a risk of contamination. The purpose of this study was to develop gelatin sponge millispheres (GSMs), a convenient and reliable agent, and characterize them in vitro.

Method: The size of GSMs was controlled by modifying the previously reported method to include the use of caprylic triglyceride and isopropanol. Analytical and microbiological tests were conducted to detect impurities (caprylic triglyceride, isopropanol, endotoxins, bacteria, and fungus). The effects of syringe volume (1.0 to $5.0 \mathrm{ml})$ and contrast media viscosity (1.6 to $13.6 \mathrm{mPa} * \mathrm{~s})$ on the in vitro injectability of GSMs through microcatheters of various inner diameters (ID) (0. 43 to $0.53 \mathrm{~mm}$ ) were examined via in-line pressure monitoring.

Results: The GSMs were found to be water-insoluble particles containing interconnected pores. The short and long diameters of the GSMs were $1.82 \pm 0.2 \mathrm{~mm}$ and $2.37 \pm 0.3 \mathrm{~mm}$, respectively. The results of tests for impurities indicated that GSMs have the general properties necessary for medical devices. The GSMs were successfully injected without clogging through a microcatheter (ID: $0.53 \mathrm{~mm}$ ) attached to a 1.0 or $2.5 \mathrm{ml}$ syringe.
\end{abstract}

Conclusion: GSMs have the basic properties and injectability necessary to be considered reliable biomaterials (eg, embolic agents).

Keywords: embolic agents, gelatin sponge millispheres, injectable scaffolds, interconnected pores, microcatheter

\section{Introduction}

Gelatin sponge is an absorbable hemostatic agent that has no toxic effect on the body, and can be absorbed completely in approximately one month. ${ }^{1}$ For these reasons, it is considered to be a safe and useful material, and has been widely used in surgical procedures for more than 60 years. $^{2}$ In addition, fragments made by hand from a sheet of gelatin sponge using a scalpel, small scissors, or a syringe have frequently been used for temporary embolization, ${ }^{3}$ in accordance with the particular indications for hepatocellular carcinoma (HCC). ${ }^{4}$ However the preparation of the fragments is burdensome and carries the risk of infecting the liver with exogenous bacteria. ${ }^{5}$ For these reasons, many clinicians have long sought reliable gelatin sponge products for use as embolic agents. ${ }^{3}$ Recently, we developed a novel method for preparing gelatin sponge millispheres (GSMs) with a diameter of $1 \mathrm{~mm}$ that would provide a reliable and convenient supply of embolic agents for HCC. ${ }^{6}$ Although the optimal size of gelatin sponge fragments for transarterial embolization (TAE) against $\mathrm{HCC}$ has been reported to be 0.5 to $1.0 \mathrm{~mm},{ }^{7}$ our surveillance indicates that sizes of $1 \mathrm{~mm}, 2 \mathrm{~mm}$, or both are most frequently requested (17 of 19) at major medical facilities in Japan. ${ }^{6}$ Therefore, in an attempt to meet the clinical demand for reliable embolic agents, a method for preparing 2-mm GSMs has been developed.

For clinical use, injectability is one of the most important properties of embolic agents; in fact, the injectability of conventionally available embolic agents has been 
documented extensively. ${ }^{3,8-10}$ Reportedly, injectability depends on the properties of the embolic agent (eg, shape, size, brand, and concentration) and those of the microcatheter (eg, inner diameter, brand, and tortuosity). It is difficult to directly compare these reports because the conditions at each institute were different, and the evaluations were conducted using sensory parameters such as "ease of injection", rather than quantifiable and reproducible methods. Thus, quantitative and objective evaluations under reproducible conditions (syringe volume, viscosity of contrast media, inner diameter of microcatheter, in-line pressure) are necessary in order to investigate clinical usage of 2-mm GSMs.

Since no such evaluation method had yet been established for embolic material, we attempted to develop the first one, and then used it to test whether 2-mm GSMs would function properly.

\section{Materials and methods}

\section{Preparation and characterization of GSMs}

GSMs were prepared using a modification of our previously reported method. ${ }^{6}$ Briefly, GSMs were prepared using airformed gelatin droplets in caprylic triglyceride (air-in-waterin-oil-type emulsions) captured in the sol-gel transition phase, followed by dehydration with isopropanol, vacuum drying, and heat treatment for cross-linking. The diameters of the droplets were made larger than those of 1-mm GSMs by using a slower (approximately 10\% to 20\% slower) speed of agitation. While being agitated in chilled isopropanol, the GSMs were sieved through punched metal containers with pore diameters twice as large as those used for 1-mm GSMs. The recovered particles were heat-treated in a vacuum drying oven at $155^{\circ} \mathrm{C}$ for four hours to cross-link the gelatin. The cross-linked particles were then dispersed in water to remove the soluble component, and the resulting particles were dispensed into vials, lyophilized (aseptic manipulation), and stored at room temperature or $5^{\circ} \mathrm{C}$ until use.

The characteristics (insolubility, diameter, microscopic observation, residual isopropanol, residual caprylic triglyceride, level of bacterial endotoxins, and sterility) of the GSMs were tested as previously reported. ${ }^{6}$

\section{Injectability of GSMs}

The injectability of GSMs through various commercially available microcatheters was tested by injecting GSMs suspended in a variety of commercially available contrast media through syringes of various volumes. The characteristics of the microcatheters and the contrast media used in this study are given in Tables 1 and 2. The following syringes were used: 1-ml BD Leur-Lok ${ }^{\mathrm{TM}}$ disposable syringe with a clear polycarbonate barrel (Becton, Dickinson and Company, Franklin Lakes, NJ, USA), Terumo 2.5-ml Luer Slip Tip Syringe (Terumo Co., Ltd., Tokyo, Japan), Terumo 5-ml Luer Slip Tip Syringe (Terumo). A round digital pressure gauge VSMC-AS-32 P-7-5 MPa-W-Q005MPGR3, 0-50 kgf/cm² (Valcom Co., Ltd., Osaka, Japan) equipped with a Shimadzu C-R7A with data processor (Shimadzu Co., Ltd., Kyoto, Japan) was connected between the syringe and catheter via a three-way stopcock (Terumo) to monitor the in-line pressure generated by the injection of GSMs through the syringe (Figure 1a).

The upper pressure limits of each syringe were calculated by averaging the maximum manual compression pressure (MC) applied by five healthy men (triplicate measurements) without using GSMs or a microcatheter. The syringe, which was loaded with saline, was connected via a three-way stopcock to the digital pressure gauge equipped with a data processor. While holding the syringe in one hand, the thumb of the other hand was used to push the plunger as hard as possible for approximately $10 \mathrm{sec}$ while monitoring the pressure. The upper pressure limit was calculated as the mean $\mathrm{MC}$ for the five three-trial averages (Equation 1).

\section{Average MC (MPa)$$
=\frac{\text { Area under the time }- \text { pressure }(\mathrm{sex} \times \mathrm{MPa}) \text { curve }}{\text { Duration of plunger push }(\mathrm{sec})}
$$

Each vial contained $80 \mathrm{mg}$ (approximately $7 \mathrm{ml}$ ) of GSMs suspended in $10 \mathrm{ml}$ of a contrast medium (all medium types were used in the trials), which was gently shaken for one minute. The contrast media were used without dilution or warming. The bubbles on the GSMs were removed by charging and discharging the syringe 10 times. One milliliter of the suspension was drawn into each syringe. The loaded syringes were connected to the catheters via a three-way stopcock equipped with a digital pressure gauge and a data processor. The catheter was placed horizontally on a lab bench, and then

Table I Characteristics of microcatheters

\begin{tabular}{|c|c|c|c|c|c|}
\hline \multirow[t]{2}{*}{ Microcatheter $^{\mathrm{a}}$} & \multicolumn{2}{|c|}{$\begin{array}{l}\text { Distal inner } \\
\text { diameter }\end{array}$} & \multicolumn{2}{|c|}{$\begin{array}{l}\text { Distal outside } \\
\text { diameter }\end{array}$} & \multirow{2}{*}{$\begin{array}{l}\text { Length } \\
(\mathrm{mm})\end{array}$} \\
\hline & (inch) & $\overline{(\mathrm{mm})}$ & $\left(\mathrm{Fr}^{\mathrm{b}}\right)$ & $\overline{(\mathrm{mm})}$ & \\
\hline FasTracker-I8MX & 0.021 & 0.53 & 2.5 & 0.84 & 150 \\
\hline Excelsior-1018 & 0.019 & 0.48 & 2.0 & 0.67 & 150 \\
\hline Excel-14 & 0.017 & 0.43 & 1.9 & 0.63 & 150 \\
\hline
\end{tabular}

Notes: aAll microcatheters manufactured by Target Therapeutics (Fremont, CA, USA)/

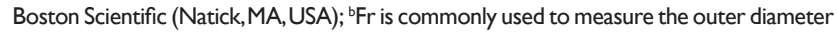
of cylindrical medical instruments, including catheters. $\mathrm{Fr}=$ diameter $(\mathrm{mm}) \times 3$. 
Table 2 Characteristics of contrast media (data from product package inserts)

\begin{tabular}{|c|c|c|c|}
\hline Contrast medium & Manufacturer & lodine content $(\mathrm{mg} \mathrm{l} / \mathrm{ml})$ & Viscosity at $37^{\circ} \mathrm{C}$ (mPa.s) \\
\hline Omnipaque I40 & $\begin{array}{l}\text { GE Healthcare (Buckinghamshire, UK)/ } \\
\text { Daiichi Sankyo (Tokyo, Japan) }\end{array}$ & 140 & 1.5 \\
\hline lopamiron 300 & Schering AG (Berlin-Wedding, Germany) & 300 & 4.4 \\
\hline Optiray 320 & $\begin{array}{l}\text { Tyco Healthcare (Mansfield, MA, USA)/ } \\
\text { Mallinckrodt (Saint Louis, MO, USA) }\end{array}$ & 320 & 5.8 \\
\hline lopamiron 370 & Schering AG (Berlin-Wedding, Germany) & 370 & 9.1 \\
\hline lomeron 400 & Bracco (Milan, Italy) & 400 & 13.6 \\
\hline
\end{tabular}

flushed with saline before each measurement. The leading edge of the catheter was immersed in saline by fixing the catheter on the wall of a petri dish with adhesive tape. While holding the syringe in one hand, the thumb of the other hand was used to push the plunger while monitoring in-line pressure. The tests were performed at room temperature. The average pressure was calculated using Equation 2.

Average pressure (MPa)

$=\frac{\text { Area under the time }- \text { pressure curve }(\sec \times \mathrm{MPa})}{\text { Duration of plunger push }(\mathrm{sec})}(2)$

When too many residual GSMs remained in the vial or syringe, additional contrast medium was added. The total volume of added contrast medium was recorded. One volume $(1,2.5$, or $5 \mathrm{ml})$ of saline was injected after the vial had been emptied to confirm that no clogging had occurred in the dead volume of the syringe and catheter line. The same catheters were used for each measurement after flushing with saline in between. Injectability was evaluated by measuring average pressure and maximum pressure during each trial (Figure 1b). When the maximum pressure was below the upper pressure limit, the conditions were judged to be "injectable." When the average or maximum pressure exceeded the upper pressure limit or the flow stopped due to clogging, the conditions were judged to be "noninjectable." The GSM trials for each syringe volume started with the largest inner diameter catheter and lowest-viscosity contrast media. There were one or three trials under each set of conditions. When the set of conditions was judged "noninjectable," the set of conditions immediately prior was repeated two more times. If at least one trial of the three was judged "noninjectable," that set of conditions was judged to be "noninjectable." In such cases, the sets of conditions using smaller inner diameter catheters or higher-viscosity contrast media were also considered "noninjectable" without being tested.

\section{Results and discussion}

The GSMs were spherical, water-insoluble particles with interconnected pores not only on the surface, but also on the interior proximal surfaces between the air spaces (Figure 2). The characteristics of the GSMs are shown in Table 3. The short and long diameters of 20 randomly extracted GSMs $(\mathrm{n}=3)$ were all $1.8 \pm 0.2 \mathrm{~mm}$ and $2.4 \pm 0.3 \mathrm{~mm}$ respectively, yielding a short/long diameter ratio of $78 \%$. The other characteristics exhibited the same basic compatibility as medical devices as the 1-mm GSMs did. ${ }^{6}$

The MC values obtained for the 1.0-, 2.5-, and 5.0-ml syringes were $2.32 \pm 0.16,0.88 \pm 0.13$, and $0.52 \pm 0.06 \mathrm{MPa}$, respectively. The relationship between plunger surface area and upper pressure limit, which was calculated as the average MC, is described in Table 4. The upper pressure limit of the smaller syringe was higher than that of the larger one, as was previously reported. ${ }^{11}$ These results are considered to be based on Pascal's law. The syringe with the smallest plunger surface area can generate the largest compression force. Therefore, although the manual force on the 1-ml syringe was slightly less, the upper pressure limit was higher than the pressures generated with larger syringes. It follows then that the $1 \mathrm{ml}$ syringe would allow easier injection. The upper pressure limits in this study are considered to be normal because previous studies reported a similar range of values [1.4 to $2.8 \mathrm{MPa}$ (200 to $400 \mathrm{psi})$ ] as "reasonable pressure" for a 1-ml syringe. ${ }^{9}$

The injectability results are given in Table 5. Injectability was influenced by the catheters' inner diameter, viscosity of the contrast media, and volume (actually, plunger surface area) of the syringes. When a 1-ml syringe was used, the injectability of GSMs through catheters with inner diameters larger than $0.48 \mathrm{~mm}$ were confirmed to be feasible. GSMs were considered to be compressed to less than $26 \%$ of their original diameter in those catheters, based on the ratio of the inner diameter of the catheter to the short diameter of the GSMs. To detect any morphological changes after passage through the catheter, the appearance of the GSMs $(n=20)$ were also examined. No remarkable changes in any of the GSMs were observed after they were passed through a catheter (FasTracker-10, Target Therapeutics [Fremont, CA, USA]/Boston Scientific [Natick, MA, USA]) with an 
a)

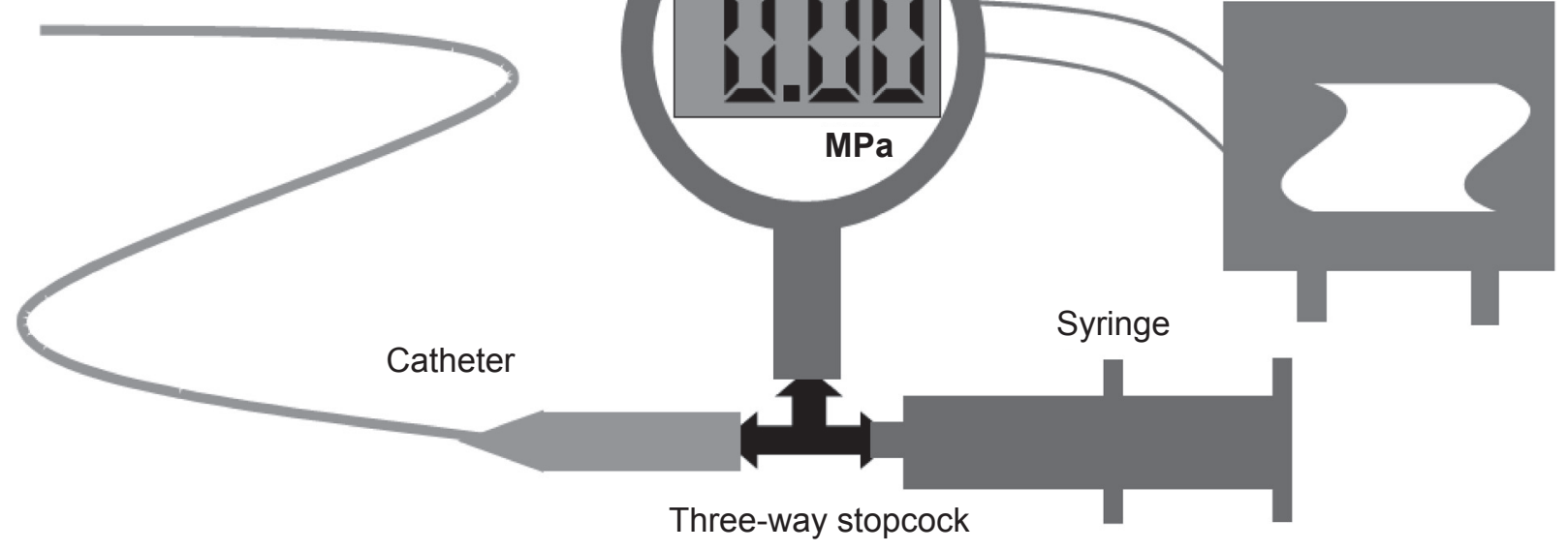

b)

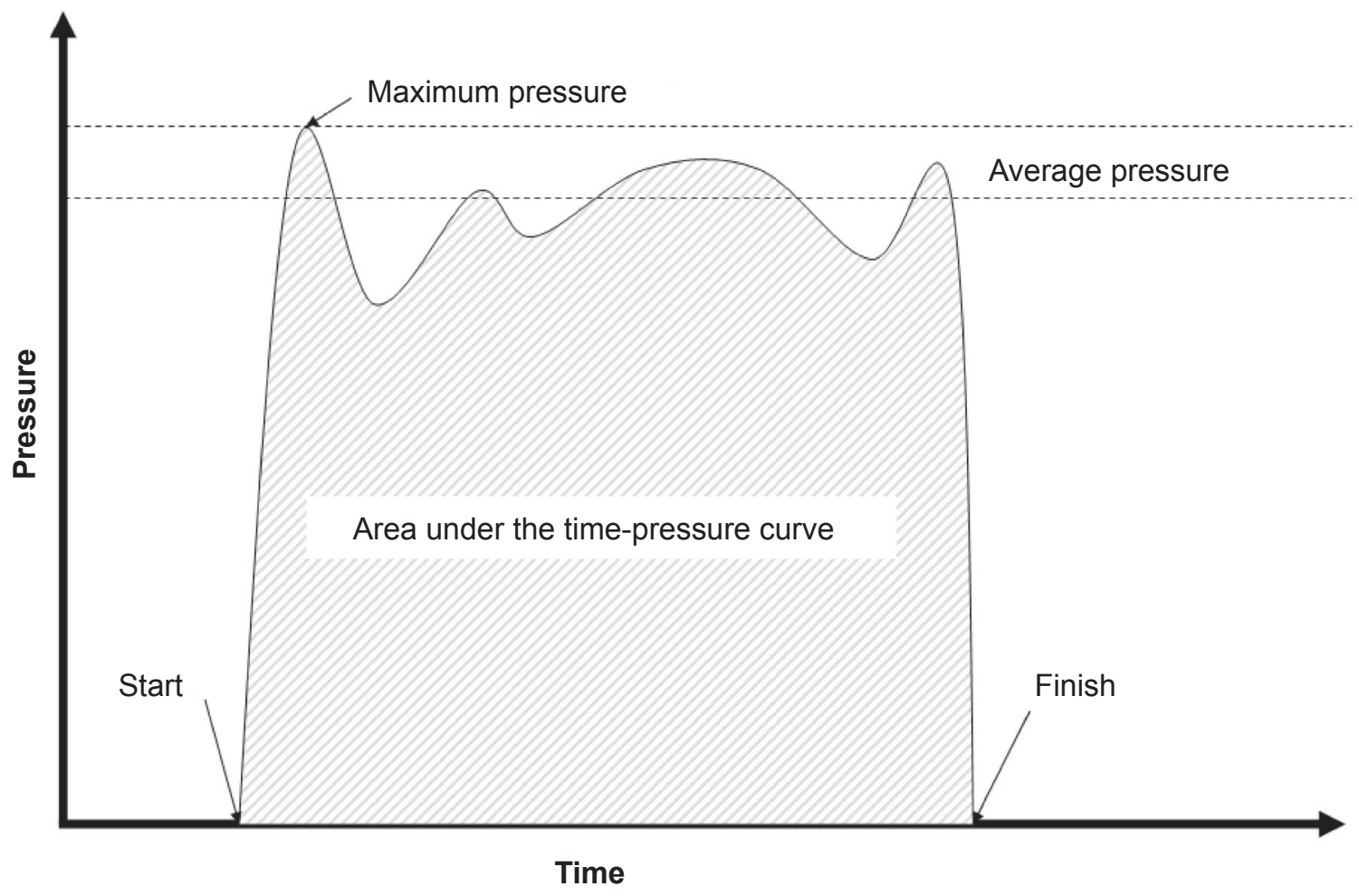

Figure I a) Pressure monitoring system. b) Time-pressure curve.

innner diameter of $0.38 \mathrm{~mm}$, which is $79 \%$ smaller than the short diameter of the GSMs themselves (unpublished data). The compression ratio was calculated to be less than $4 \%$ of the initial as determined by cross-sectional area. Particles of the conventional embolic agent (trisacryl gelatin microspheres) are not injectable through catheters with inner diameters over $23 \%$ smaller than the particle diameter without any permanent alteration of the particle. ${ }^{8}$ This differences in elasticity might be due to the sponge-like structure of the GSMs and the elasticity of the optimally cross-linked gelatin. Although conventional polyvinyl alcohol (PVA) foam also has a porous structure, 2-mm particles can be injected only on a one-by-one basis, and particle mixtures of $1.3 \mathrm{mg} / \mathrm{ml}$ cannot be injected through FasTracker-18MX. ${ }^{9}$ This noninjectability 

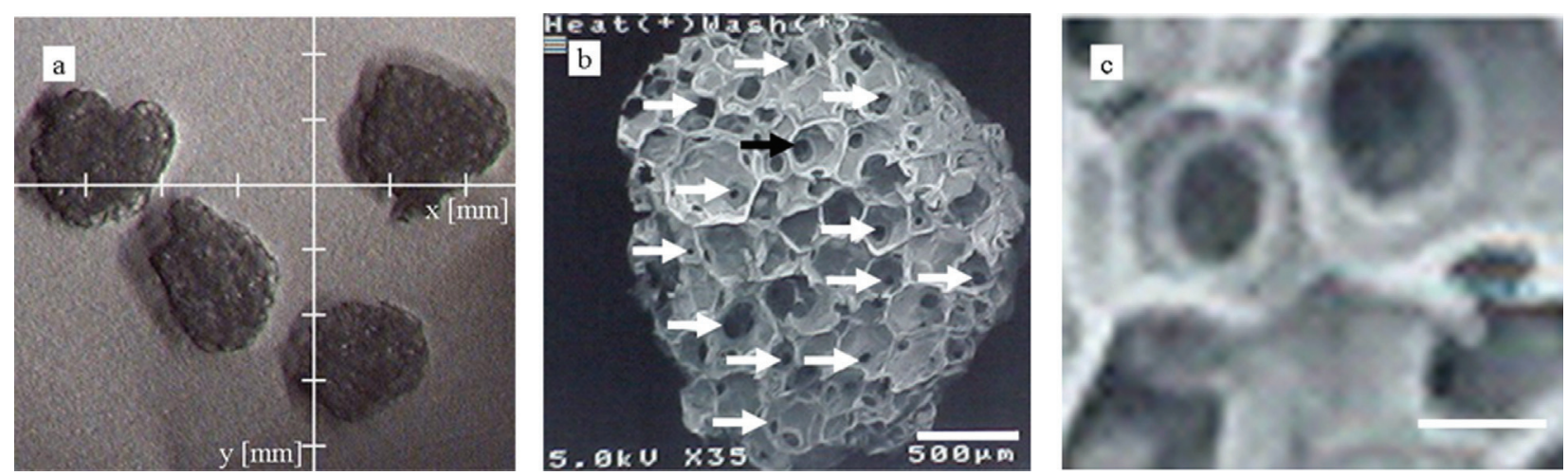

Figure 2 a) Representative optical micrograph of gelatin sponge millispheres (GSMs). Increments on the reference axes $=1,000 \mu \mathrm{m}$. b) Representative scanning electron micrograph of gelatin sponge millisphere (GSM). Cross-section of GSM. The interconnected pores are indicated by arrows. Scale marker bar in photomicrograph $=500 \mu \mathrm{m}$. c) Enlarged view of interconnected pore indicated by the black arrow in b. Scale marker bar in photomicrograph $=100 \mu \mathrm{m}$.

is considered to be due to shape. Spherical materials cause less clogging in catheters than materials of random shapes; ${ }^{8}$ even an $8 \mathrm{mg} / \mathrm{ml}$ mixture of GSMs did not cause any clogging under similar conditions (Luer-lock syringes equipped with a microcatheter (ID: $0.53 \mathrm{~mm}$ ). Although cubes of conventional gelatin sponges (eg, Gelfoam ${ }^{\circledR}$ [Pfizer Inc., New York, NY, USA] or Spongel ${ }^{\circledR}$ [Astellas Pharma Inc., Tokyo, Japan], approximately $1-1.5 \times 1-1.5 \mathrm{~mm}$ ) dispersed in saline have been injected through a microcatheter (ID: $0.56 \mathrm{~mm}$ ) using a 2.5-mL syringe, ${ }^{3}$ the injectability of GSMs can be considered the same or better since their shape and porosity offers even less resistance during the injection process. In recent years the gelatin sponge GelitaSpon IR Sponge GS-322 (Gelita Medical B.V., Amsterdam, The Netherlands) has become commercially available in Europe; however, this is also cubic in shape $(3 \times 2 \times 2 \mathrm{~mm})$. A larger caliber chatheter (2.7 to 5.0 Fr catheter [ID: 0.65 to $1.2 \mathrm{~mm}$ or more]) is still recommended for use of this product.

Table 3 Characteristics of gelatin sponge millispheres (GSMs)

\begin{tabular}{|c|c|c|}
\hline Characteristic & $2 \mathrm{~mm}-\mathrm{GSMs}$ & $(1 \mathrm{~mm}-\mathrm{GSM})^{\mathrm{a}}$ \\
\hline Short diameter $(\mathrm{mm})$ & $1.8 \pm 0.2$ & $\mathrm{I} . \mathrm{I} \pm 0.2$ \\
\hline Long diameter (mm) & $2.4 \pm 0.3$ & $1.3 \pm 0.2$ \\
\hline $\begin{array}{l}\text { Short/long diameter } \\
\text { ratio }\end{array}$ & $78 \%$ & $86 \%$ \\
\hline Residual isopropanol & $\mathrm{BLD}^{\mathrm{b}}$ & BLD \\
\hline $\begin{array}{l}\text { Residual caprylic } \\
\text { triglyceride }\end{array}$ & $0.10 \% \pm 0.08 \%(w / w)$ & $0.16 \% \pm 0.04 \%(w / w)$ \\
\hline Bacterial endotoxins & $\mathrm{BLD}^{\mathrm{c}}$ & BLD \\
\hline Sterility & Sterile $^{d}$ & Sterile \\
\hline
\end{tabular}

Notes: ${ }^{a}$ Yamashita ${ }^{6}$; ${ }^{b}$ Below the limit of detection, $<0.05 \%$ (w/w); ; Below the limit of detection, $<0.025 \mathrm{EU} / \mathrm{ml} ;{ }^{d} \mathrm{No}$ bacterial or fungal growth was detected during GSM sterility testing using the direct method. ${ }^{6}$
Increases in the viscosity of the contrast media caused increases in the maximum pressure required during passage or clogging in the catheter. This can be explained by increased resistance in the catheter, as represented by Equation 3,

$$
R=\frac{128 \eta L}{\pi d^{4}}
$$

This equation was derived from Hagen-Poiseuille's Equation (4),

$$
\Delta P=\frac{128 \eta L Q}{\pi d^{4}}
$$

where: $\Delta P$ is the pressure drop, $L$ is the length of the tube, $\eta$ is the dynamic viscosity, $Q$ is the volumetric flow rate, $d$ is the diameter, and $\pi$ is the mathematical constant. As electricity was originally understood to be a kind of fluid, this hydraulic analogy is still conceptually useful. Then Hagen-Poiseuille's law corresponds to Ohm's law for electrical circuits $(V=I R)$, where the pressure drop $(\Delta P)$ is analogous to the voltage $(V)$, and the voluminal flow rate $(Q)$ is analogous to the current $(I){ }^{12}$ The resistance, then, is represented by Equation 3. Although the mixture of GSMs with contrast medium is a suspension (ie, not completely homogeneous) and the flow may not be steady, the Hagen-Poiseuille equation was used for simplification. This means that the resistance $(R)$ is proportional to the viscosity of the contrast medium $(\eta)$ and the length of the catheter $(L)$, and is in inverse proportion to the inner diameter of catheter $(d)$ raised to the fourth power. ${ }^{13}$ The ideal pressure loss calculated by FasTracker-18MX is considered to be less than that by Excelsior1018 (1.5-fold) or Excel-14 (2.3-fold), based on the difference in diameter (Table 1). The ideal pressure drop caused by the Omnipaque 140 is considered 
Table 4 Upper pressure limit of syringe

\begin{tabular}{llll}
\hline $\begin{array}{l}\text { Syringe } \\
\text { volume }(\mathbf{m l})\end{array}$ & $\begin{array}{l}\text { Plunger surface } \\
\text { area }\left(\mathbf{m m}^{2}\right) \text { (Ratio) }\end{array}$ & $\begin{array}{l}\text { Upper pressure limit } \\
(\text { Mpa) (Inverse ratio) }\end{array}$ & Force (N) \\
\hline 1.0 & $17.3(1.0)$ & $2.32(1.0)$ & 40.3 \\
2.5 & $62.9(3.6)$ & $0.88(2.6)$ & 55.4 \\
5.0 & $134(7.7)$ & $0.52(4.5)$ & 69.4 \\
\hline
\end{tabular}

to be less than Iopamiron 300 (2.9-fold), Optiray 320 (3.9fold), Iopamiron 370 (6.1-fold), and Iomeron 400 (9.1-fold) based on the difference in viscosity (Table 2).

Lower fluid viscosity $(\eta)$ can result in easier injection, which can also be influenced by the brand of contrast medium, ${ }^{14}$ its temperature ${ }^{15}$ and dilution ratio, ${ }^{16}$ and the concentration of particles. ${ }^{9}$ Microcatheters with shorter lengths (L) and larger inner diameters (radius [a]) are recommended for easier injection. It has been reported that the difference in the brand of catheter can also affect the injectability of conventional embolic agents. ${ }^{9,10}$ The difference may be caused by the inherent properties of each catheter. ${ }^{17}$ Although no ruptures or leaks were observed in the microcatheters tested during our study, the manufacturer's suggested operating pressure limit for each microcatheter is less than most of the pressures tested. Since some people could generate more than $4.8 \mathrm{MPa}$ (700 psi) of pressure with a 1-ml syringe during the trials, microcatheters guaranteed to withstand high pressure are preferable for clinical use in order to lessen the risk of rupture in the body of a patient. ${ }^{9,17}$

Theoretically, a slower injection rate (flow rate [Q]) is recommended for easier injection. Given that Barr and colleagues ${ }^{9}$ observed that slow infusion $(0.2$ to $0.4 \mathrm{ml} / \mathrm{sec})$ caused greater occlusion of PVA foam particles than rapid injection, and our study did not clarify the relationship between flow rate and injectability based on manual injection, further investigation into the optimal flow rate is needed. Barr and colleagues suggested that periodic cleaning of the catheter hub during long procedures may be useful for preventing clumping of particles in that region. ${ }^{9}$

For clinical use, the conditions mentioned above should be optimized from a therapeutic point of view. If there is some allowance in the conditions, they can be modified for easier injection. Such modifications can be useful because the resistance may increase due to catheter tortuosity or other factors (environment around catheter) during clinical use.

In our previous report, ${ }^{6} 1-\mathrm{mm}$ GSMs were prepared and characterized. These smaller GSMs were confirmed to be injectable through microcatheters with an ID as small as $0.28 \mathrm{~mm}(0.011 \mathrm{inch})$ using a $1-\mathrm{ml}$ syringe at a pressure below the upper pressure limit (unpublished data).

We consider GSMs to be useful not only as therapeutic embolization devices, but also for fundamental applications in other biotechnology areas (eg, tissue engineering), ${ }^{18}$ given that their unique properties (ie, injectability, interconnectedpore structure, biocompatibility, and sterility) render them suitable as biomaterials (eg, cell scaffolds of cells). In fact, the use of porous gelatin spheres of $0.5-\mathrm{mm}$ or less as scaffolds has already been reported. ${ }^{19}$

\section{Conclusion}

GSMs, spherical particles with interconnected pores, were prepared in the 2-mm size range. The results of analytical and microbiological testing for impurities (caprylic triglyceride, isopropanol, endotoxins, bacteria, and fungus) indicated that these GSMs have the general properties necessary

Table 5 Injectability of GSMs under various condition

\begin{tabular}{|c|c|c|c|}
\hline \multirow{2}{*}{$\begin{array}{l}\text { Contrast media } \\
\text { viscosity (mPa.s) }\end{array}$} & \multicolumn{3}{|c|}{ Catheter inner diameter $(\mathrm{mm})$} \\
\hline & FasTracker-18MX (0.53) & Excelsior $-1018(0.48)$ & Excel -I4 (0.43) \\
\hline Omnipaque I40 (I.5) & all & $\mathrm{I} \mathrm{ml}$ & none \\
\hline lopamiron 300 (4.4) & $\mathrm{I}$ and $2.5 \mathrm{ml}$ & $\mathrm{I} \mathrm{ml}$ & none \\
\hline Optiray 320 (5.8) & $\mathrm{I}$ and $2.5 \mathrm{ml}$ & $\mathrm{I} \mathrm{ml}$ & none \\
\hline lopamiron 370 (9.1) & $\mathrm{I}$ and $2.5 \mathrm{ml}$ & $\mathrm{I} \mathrm{ml}$ & none \\
\hline lomeron 400 (I3.6) & $\mathrm{I}$ and $2.5 \mathrm{ml}$ & $\mathrm{I} \mathrm{ml}$ & none \\
\hline
\end{tabular}

Notes: “All”: injectability was confirmed for the I-, 2.5- and 5-ml syringes; “I and 2.5 ml”: injectability was confirmed only for the I- and 2.5-ml syringes, but not the 5-ml; “I ml”: Injectability was confirmed only for the I-ml syringe, but not the 2.5 - or 5-ml syringe; “none": injectability was NOT confirmed for the I-, 2.5 - or 5 ml syringes. 
for use as medical devices. GSMs are injectable through conventional-sized microcatheters (ID: $0.53 \mathrm{~mm}$ ) using a 1.0 - or $2.5-\mathrm{ml}$ syringe with reasonable finger pressure.

\section{Acknowledgments}

The authors would like to thank Dr Ryusaku Yamada (MD, PhD), professor emeritus of Osaka City University, and Dr Hironobu Nakamura (MD, PhD), professor of Osaka University Graduate School of Medicine, for coordinating our surveillance studies at major medical facilities in Japan. The authors report no conflicts of interest in this work.

\section{References}

1. Correll JT, Prentice HR, Wise EC. Biologic investigations of a new absorbable sponge. Surg Gynecol Obstet. 1945;81:585-589.

2. Jenkins HP, Janda R, Clarke J. Clinical and experimental observations on the use of gelatin sponge or foam. Surgery. 1946;20:124-132.

3. Katsumori T, Kasahara T. The size of gelatin sponge particles: differences with preparation method. Cardiovasc Intervent Radiol. 2006;29:1077-1083.

4. Yamada R, Kishi K, Sonomura T, et al. Transcatheter arterial embolization in unresectable hepatocellular carcinoma. Cardiovasc Intervent Radiol. 1990;13:135-139.

5. Yokoi Y, Suzuki S, Sakaguchi T, et al. Subphrenic abscess formation following superselective transcatheter chemoembolization for hepatocelluar carcinoma. Radiat Med. 2002;20:45-49.

6. Yamashita N, Izumikawa S, Takagi A, Arakawa H, Wakasawa T, Maruyama A. Preparation and characterization of gelatin sponge millispheres from air-in-water-in-oil-type emulsions. J Mater Sci Mater Med. 2008;In Press, DOI: 10. 1007/s10856-008-3681-1.

7. Sonomura T. Optimal size of embolic material in transcatheter arterial embolization of the liver [Article in Japanese]. Nippon Igaku Hoshasen Gakkai Zasshi, 1994;54:489-499.
8. Laurent A, Beaujeux R, Wassef M, et al. Trisacryl gelatin microspheres for therapeutic embolization, I: Development and in vitro evaluation. Am J Neuroradiol. 1996;17:533-540.

9. Barr JD, Lemley TJ, Petrochko CN. Polyvinyl alcohol foam particle sizes and concentrations injectable through microcatheters. $J$ Vasc Interv Radiol. 1998;9(1 Pt 1):113-118.

10. Lewis AL, Adams C, Busby W, et al. Comparative in vitro evaluation of microspherical embolisation agents. J Mater Sci Mater Med. 2006;17:1193-1204.

11. Abele JE. Balloon catheters and transluminal dilatation: Technical considerations. Am J Roentgenol. 1980;135:901-906.

12. Sutera SP, Skalak R. The history of Poiseuille's law. Ann Rev Fluid Mech. 1993;25:1-20.

13. Samett EJ. Basic fluid dynamics principles - Application to percutaneous intervention. eMedicine Clinical Knowledge Base [serial on the Internet]. New York: WebMD, Inc.; c1996-2008. Updated 2004 Mar 31; [cited 2008 Oct.] 12.Available from: http://www.emedicine. com/radio/topic867.htm.

14. Jakobsen JA. Physiological effects of contrast media for use in multidetector row computed tomography. Eur J Radiol. 2007;62:14-25.

15. Herts BR, Cohen AH, McInroy B, et al. Power injection of intravenous contrast material through central venous catheters for $\mathrm{CT}$ : in vitro evaluation. Radiology. 1996;200:731-735.

16. Yamauchi T, Furui S, Suzuki S, et al. A simple method to estimate the optimum iodine concentration of contrast material through microcatheters: hydrodynamic calculation with spreadsheet software. Phys Med Biol. 2008;53:1385-1395.

17. Sadaoka S. Comparative study of the physical properties of microcatheters frequently used for abdominal IVR [Article in Japanese]. Nippon Igaku Hoshasen Gakkai Zasshi. 1999;59:867-876.

18. Rohanizadeh R, Swain MV, Mason RS. Gelatin sponges $\left(\right.$ Gelfoam $\left.^{\circledR}\right)$ as a scaffold for osteoblasts. J Mater Sci Mater Med. 2008;19:1173-1182.

19. Nilsson K, Buzsaky F, Mosbach K. Growth of anchorage-dependent cells on macroporous microcarriers. Nat Biotechnol. 1986;4:989-990. 
